\title{
Modeling of torsional vibration induced by extension-twisting coupling of anisotropic composite laminates with piezoelectric actuators
}

\author{
Mei-Ling Zhu ${ }^{1,2}$, Shi-Wei Ricky Lee ${ }^{1}$, Hing-Leung $\mathbf{L i}^{1}$, \\ Tong-Yi Zhang ${ }^{1}$ and Pin Tong ${ }^{1}$ \\ ${ }^{1}$ Department of Mechanical Engineering, The Hong Kong University of Science \\ and Technology, Clear Water Bay, Kowloon, Hong Kong \\ ${ }^{2}$ Institute of Vibration Engineering Research, Nanjing University of Aeronautics \\ and Astronautics, Nanjing, 210016, People's Republic of China
}

Received 20 December 2000, in final form 26 October 2001

Published 6 February 2002

Online at stacks.iop.org/SMS/11/55

\begin{abstract}
In this paper we present a dynamic analytical model for the torsional vibration of an anisotropic piezoelectric laminate induced by the extension-twisting coupling effect. In the present approach, we use the Hamilton principle and a reduced bending stiffness method for the derivation of equations of motion. As a result, the in-plane displacements are not involved and the out-of-plane displacement of the laminate is the only quantity to be calculated. Therefore, the proposed method turns the twisting of a laminate with structural coupling into a simplified problem without losing its features. We give analytical solutions of the present model with harmonic excitation. A parametric study is performed to demonstrate the present approach.
\end{abstract}

\section{Introduction}

Due to the demand for advanced performance in modern engineering systems, smart materials and structures have been one of the main research and development areas for a decade. One of the major characteristics of smart materials and structures is their adaptability. In order to achieve this feature, smart materials and structures must possess simultaneous sensing and actuation capabilities. Therefore, the integration of sensing/actuation and load-bearing capacity is the main subject of research on smart materials and structures. Piezoelectric materials show relatively good performances in both of these functions. Their fast response, high-energy density and the fact that these materials generate no magnetic field make them widely utilized in actuation devices and vibration control. Sunar and Rao [1] have provided a detailed review of the recent advances in the sensing and control of flexible structures via piezoelectric material technology.

Surface-bonded and embedded piezoelectric sensors and actuators in composite laminates are commonly used smart structures. In recent decades, a considerable number of laminate theories, analytical approaches, numerical solutions and computational models have been reported for the analysis of laminates and structures with piezoelectric actuators or sensors. In addition, the finite element method (FEM) has been established as a powerful numerical technique, which provides solutions to engineering problems and is widely used in modern engineering designs and analyses. Saravanos and Heyliger [2] and Tani et al [3] have provided detailed reviews of published work in these areas. Especially in modeling, Saravanos and Heyliger [2] have classified the mechanics and computational models for the laminated piezoelectric beam, plates and shells into induced strain models, equivalent single-layer models, coupled single-layer model, layerwise models and exact solutions, and have considered the thermal effect and nonlinear effect models. Their reviews are very detailed and complete. In this paper, we are not going to review these again, as we are mainly concerned with the modeling of the coupling vibration of laminated composite structures with piezoelectric actuators. 
Mathematical models of the coupling vibration of laminated composite structures with piezoelectric actuators are available in the literature. For example, Aldraihem and Wetherhold [4] have proposed a shear-deformation beam theory to model the bending and twisting coupling vibration in laminated beams with a PZT/epoxy actuator. Mass coupling and stiffness coupling are considered in their model. Dimitriadis et al [5] studied a two-dimensional model of a piezoelectric patch bonded to the surface of a rectangular plate. Wang and Rogers [6] also investigated a model in the same way as Dimitriadis et al, in order to determine the equivalent forces of a piezoelectric patch in a laminate plate. Shen [7] studied a mathematical model of intelligent constrained-layer (ICL) composite beams by integrating existing ICL plate models. The proposed ICL composite beam model is capable of explaining the coupling between bending, axial and torsional vibrations of the beams. Yang and Bian [8] conducted vibration suppression experiments on composite laminates using an embedded piezoelectric sensor and actuator. Experiments have shown that both bending and torsional vibrations of the structure can be effectively reduced by velocity feedback. Park et al [9] presented a static elastic model for isotropic beams of bending and torsion with induced-strain actuators. Lee and Chan [10] studied the actuation mechanism of a torsional motion induced by extension-twisting coupling for laminated composite beams with embedded piezoelectric materials. Following this, Lee and Sun [11] presented a static analysis of bending, twisting and shearing of a beam with a piezoelectric film. A one-dimensional beam theory was developed to model the piezoelectric composite beam. More recently, Lee and Li [12], using the actuation mechanism investigated in [13], have designed a rotary motor driven by an anisotropic composite lamina with piezoceramics as the actuator, and studied the vibration control and computational modeling using the finite element analysis (FEA) method. The structure of the driving element was a three-layer laminated plate. Due to the material anisotropy and antisymmetric configuration, torsional vibration can be induced from in-plane strain actuation in the structure, and a rotary motor can be implemented. In order to design the motor structure and predict the motor performance, we need to construct a mathematical model of the motor, including the longitudinal and torsional vibrations of the laminate and the rotating motion of the rotor. However, it is difficult to insert a present dynamic model, for example, commercial FEA software, in the mathematical model of the motor. Therefore, an investigation by analytical modeling for torsional vibration induced by extension-twisting coupling is necessary.

In this paper we present a dynamic analytical model of torsional vibration induced by extension-twisting coupling of anisotropic composite laminates with piezoelectric actuators using a non-conventional method. In addition, the Hamilton principle and the classical plate theory are used for the derivation of the model. We give the analytical solution of the model in the case of harmonic excitation. Some comparisons are drawn with a more accurate FEA model.

\section{Analytical model}

In this section, we consider a thin laminate, as shown in figure 1, composed of a PZT layer and two layers of composite

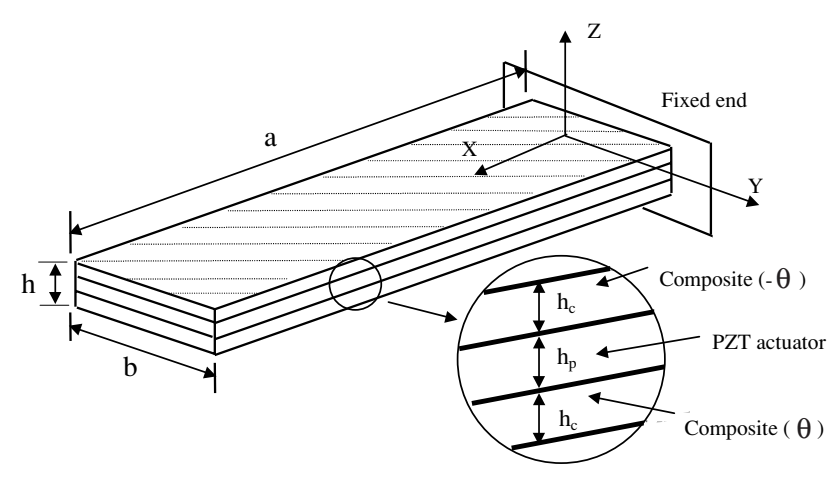

Figure 1. Configuration and dimensions of the plate under investigation.

laminae with arbitrary in-plane orientation. The laminae are made of graphite/epoxy composite laminae that have anisotropy and antisymmetric configuration with the center layer, while the center layer is a piezoelectric material, serving as an actuator. Due to the material anisotropy and the antisymmetric configuration, torsional vibration can be induced through the in-plane strain actuated by the piezoelectric layer.

The composite laminate has a length of $a$ (along the $x$-axis) and a width of $b$ (along the $y$-axis). The thickness and density of the graphite/epoxy layer is $h_{c}$ and $\rho_{c}$. The piezoelectric layer is polarized in the thickness direction and has a thickness of $h_{p}$ and a density of $\rho_{p}$. The composite laminate is subjected to an in-plane piezoelectric force without external forces and moments applying to the laminate. When an alternating electric voltage is applied to the piezoelectric layer across its thickness, the laminate will perform torsional vibration.

Due to the material anisotropy and structural extensiontwisting coupling, it may be rather difficult to achieve closeform analytical solutions of extension-twisting coupling for the aforementioned composite laminate. In the present study, we adopt a non-conventional method to present an analytical model of the laminate. In addition, the Hamilton principle and the classical plate theory are used for the derivation of the model.

Generally speaking, a conventional method to derive vibration equation requires assumed harmonic solutions of the mid-plane displacements. The assumed harmonic solutions take the form

$$
\begin{aligned}
u_{0}(x, y, t) & =\mathrm{e}^{\mathrm{i} \omega t} U(x, y) \\
v_{0}(x, y, t) & =\mathrm{e}^{\mathrm{i} \omega t} V(x, y) \\
w_{0}(x, y, t) & =\mathrm{e}^{\mathrm{i} \omega t} W(x, y)
\end{aligned}
$$

where

$$
\begin{gathered}
U(x, y)=\sum_{m=1}^{M 1} \sum_{n=1}^{N 1} E_{m n} U_{m n}(x, y) \\
V(x, y)=\sum_{m=1}^{M 2} \sum_{n=1}^{N 2} F_{m n} V_{m n}(x, y) \\
W(x, y)=\sum_{m=1}^{M 3} \sum_{n=1}^{N 3} H_{m n} W_{m n}(x, y)
\end{gathered}
$$


in which $E_{m n}, F_{m n}$ and $H_{m n}$ are undetermined coefficients, and the functions $U_{m n}(x, y), V_{m n}(x, y)$ and $W_{m n}(x, y)$ are known shape functions that satisfy boundary conditions. The key to the method is to find the functions of $U_{m n}(x, y)$, $V_{m n}(x, y)$ and $W_{m n}(x, y)$ that satisfy the boundary conditions. However, in the cantilever plate we are studying, extensiontwisting coupling may cause considerable difficulty in making assumptions about the in-plane solutions to boundary value problems.

Now we consider a non-conventional method. This method neglects the in-plane inertia and uses the reduced bending stiffness matrix. This matrix was mentioned in the book [14] and was first discussed by Reissner and Starsky [15]. Here, our aims for the method are that it is not necessary to find in-plane displacements in deriving the equations of motion, but that it is only necessary to find an out-of-plane displacement of the laminate.

\subsection{Theoretical foundation}

For an isothermal process, the generalized Hamilton principle to include electroelasticity is written, by Parton and Kudryavtsev [16], as

$$
\begin{aligned}
& \int_{t_{0}}^{t_{1}} \int_{V}(\delta H-\delta K) \mathrm{d} v \mathrm{~d} t=\int_{t_{0}}^{t_{1}}\left[\int_{V} \boldsymbol{G} \delta \boldsymbol{u} \mathrm{d} v\right. \\
& \left.\quad+\int_{S}\left(\boldsymbol{f} \delta \boldsymbol{u}+q_{i} \delta \phi\right) \mathrm{d} s\right] \mathrm{d} t
\end{aligned}
$$

where $t_{0}$ and $t_{1}$ are two arbitrary instants, $\delta$ is the variation operator, $H$ is the electric enthalpy, $K$ is the kinetic energy, $G$ is the body force, $f$ is the traction along the boundary surface, $q_{i}$ is the electric charge per unit area on the boundary surface, $\boldsymbol{u}$ is the displacement vector, $\phi$ is the electric potential, $V$ is the entire domain of the volume, and $S$ is the boundary of the domain.

The electric enthalpy $H$ is defined by the equation as

$$
H(\{S\},\{E\})=\frac{1}{2}\left[\{\sigma\}^{T}\{S\}-\{D\}^{T}\{E\}\right]
$$

where $H$ is the function of the strain and the electric field, $\{\sigma\}$ is the stress vector, $\{S\}$ is the mechanical strain vector, $\{D\}$ is the electric displacement vector, and $\{E\}$ is the electric field vector.

The kinetic energy is defined as

$$
K=\frac{1}{2}\{\boldsymbol{u}\}^{T} \rho\{\boldsymbol{u}\}
$$

where $\{\boldsymbol{u}\}$ is the velocity vector of the laminate, $\{\boldsymbol{u}\}=$ $\left\{\begin{array}{lll}u & v & w\end{array}\right\}^{T}$ is the displacement vector, and $\rho$ is the density of the lamina.

\subsection{Constitutive equation and analysis}

The constitutive equations in the principal material coordinates for piezoelectric material under plane stress are

$$
\begin{aligned}
\{\sigma\} & =\left[\bar{Q}_{p}\right]\{S\}-[e]^{T} E_{3} \\
D_{3} & =[e]\{S\}+\varepsilon_{p} E_{3}
\end{aligned}
$$

where $\{\sigma\}=\left\{\sigma_{x} \sigma_{y} \sigma_{x y}\right\}^{T},\left[\bar{Q}_{p}\right]$ is the elastic constant matrix of the piezoelectric material, $\{S\}=\left\{\varepsilon_{x} \varepsilon_{y} \varepsilon_{x y}\right\}^{T},[e]$ is the piezoelectric constant matrix, and $\varepsilon_{p}$ is the dielectric constant. Since the structure under consideration is a thin laminated plate, the poling direction of the piezoelectric material is in the $z$-direction, and an external voltage is only applied in the poling direction. Therefore, the electric field $\{E\}$ and the electric displacement $\{D\}$ in equation (4) are assumed to be non-zero in the $z$-direction only. As a result, $\{E\}=E_{3}$, $\{D\}=D_{3}$, the piezoelectric constant matrix is $[e]=$ $\left[e_{31} e_{31} 0\right]$.

The relation between the electric field $E_{3}$ and the electric potential $\phi$ is

$$
E_{3}=-\frac{\partial \phi}{\partial z} .
$$

The relation between the applied voltage $V_{3}(t)$ and the electric potential $\phi$ on the electrodes is defined as $\phi=V_{3}(t)$.

The constitutive equations for the graphite/epoxy composite material are

$$
[\sigma]=\left[\bar{Q}_{c}\right][S]
$$

where $\left[\bar{Q}_{c}\right]$ is the transformed reduced stiffness matrix of the graphite/epoxy lamina. In terms of the reduced stiffness matrix $[Q]$ and the layer angle $\alpha$ between the positive direction, $x$, of the laminate and the fibre direction of the lamina, the transformed reduced stiffness matrix can be expressed as

$$
\left[\bar{Q}_{c}\right]=\left[P_{t}\right][Q]\left[P_{t}\right]^{T}
$$

where

$$
\begin{gathered}
{[Q]=\left[\begin{array}{lll}
Q_{11} & Q_{12} & Q_{16} \\
Q_{12} & Q_{22} & Q_{26} \\
Q_{16} & Q_{26} & Q_{66}
\end{array}\right]} \\
{\left[P_{t}\right]=\left[\begin{array}{ccc}
\cos ^{2} \alpha & \sin ^{2} \alpha & 2 \cos \alpha \sin \alpha \\
\sin ^{2} \alpha & \cos ^{2} \alpha & -2 \cos \alpha \sin \alpha \\
-\cos \alpha \sin \alpha & \cos \alpha \sin \alpha & \cos ^{2} \alpha-\sin ^{2} \alpha
\end{array}\right]}
\end{gathered}
$$

in which

$$
Q_{i j}=c_{i j}-\frac{c_{i 3} c_{j 3}}{c_{33}} \quad i=1,2,6 \quad j=1,2,6
$$

where $c_{i j}$ is the elastic constant of material.

In particular, the constitutive equations for a piezoelectric anisotropic composite laminate with antisymmetric configuration is

$\left\{\begin{array}{c}N_{x} \\ N_{y} \\ N_{x y} \\ \cdots \\ M_{x} \\ M_{y} \\ M_{x y}\end{array}\right\}=\left[\begin{array}{ccc:ccc}A_{11} & A_{12} & 0: 0 & 0 & B_{16} \\ A_{12} & A_{22} & 0: 0 & 0 & B_{26} \\ 0 & 0 & A_{66}: B_{16} & B_{26} & 0 \\ \cdots & \ldots & \cdots: \cdots & \cdots & \ldots \\ 0 & 0 & B_{16}: D_{11} & D_{12} & 0 \\ 0 & 0 & B_{26}: D_{12} & D_{22} & 0 \\ B_{16} & B_{26} & 0: 0 & 0 & D_{66}\end{array}\right]\left\{\begin{array}{c}\varepsilon_{x}^{0} \\ \varepsilon_{y}^{0} \\ \varepsilon_{x y}^{0} \\ x y \\ \kappa_{x}^{0} \\ \kappa_{y}^{0} \\ \kappa_{x y}^{0}\end{array}\right\}-\left[\begin{array}{c}N_{x}^{p} \\ N_{y}^{p} \\ N_{x y}^{p} \\ \cdots y \\ M_{x}^{p} \\ M_{y}^{p} \\ M_{x y}^{p}\end{array}\right]$.

Equation (13) shows that, when the composite laminate has the aforementioned configuration, the coupling between the bending and twisting does not exist in the laminate because $D_{16}$ and $D_{26}$ are zero. However, the extension-twisting coupling does exist, because $B_{16}$ and $B_{26}$ are non-zero. For simplicity of analysis, equation (13) is written in the matrix form

$$
\left[\begin{array}{l}
N \\
M
\end{array}\right]=\left[\begin{array}{ll}
A & B \\
B & D
\end{array}\right]\left[\begin{array}{l}
\varepsilon^{0} \\
\kappa^{0}
\end{array}\right]-\left[\begin{array}{l}
N^{p} \\
M^{p}
\end{array}\right]
$$




$$
\begin{aligned}
& \text { where } \\
& N=\left\{N_{x}, N_{y}, N_{x y}\right\}^{T}=\int_{-h / 2}^{h / 2}\left(\sigma_{x}^{(k)}, \sigma_{y}^{(k)}, \sigma_{x y}^{(k)}\right)^{T} \mathrm{~d} z \\
& N^{p}=\left\{N_{x}^{p}, N_{y}^{p}, N_{x y}^{p}\right\}^{T}=\int_{-h_{p} / 2}^{h_{p} / 2}[e]^{T} E_{3} \mathrm{~d} z \\
& M=\left\{M_{x}, M_{y}, M_{x y}\right\}^{T}=\int_{-h / 2}^{h / 2}\left(\sigma_{x}^{(k)}, \sigma_{y}^{(k)}, \sigma_{x y}^{(k)}\right)^{T} z \mathrm{~d} z \\
& M^{p}=\left\{M_{x}^{p}, M_{y}^{p}, M_{x y}^{p}\right\}^{T}=\int_{-h_{p} / 2}^{h_{p} / 2}[e]^{T} E_{3} z \mathrm{~d} z \\
& \varepsilon^{0}=\left\{\varepsilon_{x}^{0}, \varepsilon_{y}^{0}, \varepsilon_{x y}^{0}\right\}^{T}=\left\{\frac{\partial u_{0}(x, y, t)}{\partial x}, \frac{\partial v_{0}(x, y, t)}{\partial y},\right. \\
& \left.\frac{\partial u_{0}(x, y, t)}{\partial y}+\frac{\partial v_{0}(x, y, t)}{\partial x}\right\}^{T} \\
& \kappa^{0}=\left\{\kappa_{x}^{0}, \kappa_{y}^{0}, \kappa_{x y}^{0}\right\}^{T}=\left\{-\frac{\partial^{2} w_{0}(x, y, t)}{\partial x^{2}},-\frac{\partial^{2} w_{0}(x, y, t)}{\partial y^{2}},\right. \\
& \left.-2 \frac{\partial^{2} w_{0}(x, y, t)}{\partial x \partial y}\right\}^{T} \\
& \left(A_{i j}, B_{i j}, D_{i j}\right)=\int_{-h / 2}^{h / 2} Q_{i j}\left(1, z, z^{2}\right) \mathrm{d} z
\end{aligned}
$$

in which the superscript $(k)$ denotes the $k$ th layer, $u_{0}(x, y, t)$, $v_{0}(x, y, t)$ and $w_{0}(x, y, t)$ are mid-plane displacements, and

$$
\begin{aligned}
& \varepsilon_{x}=\varepsilon_{x}^{0}+z \kappa_{x}^{0} \\
& \varepsilon_{y}=\varepsilon_{y}^{0}+z \kappa_{y}^{0} \\
& \varepsilon_{x y}=\varepsilon_{x y}^{0}+z \kappa_{x y}^{0} .
\end{aligned}
$$

\subsection{Integration of electric enthalpy}

Substituting equations (6) and (8) into equation (4), and letting $\left\{\sigma_{m}\right\}=\left[\bar{Q}_{p}\right]\{S\}$ or $\left[\left\{\sigma_{m}\right\}\left[\bar{Q}_{c}\right]\{S\}\right.$, the electric enthalpy for the laminate becomes

$H=\left\{\begin{array}{l}\frac{1}{2}\left(\left\{\sigma_{m}\right\}^{T}\{S\}-2 E_{3}[e]\{S\}-\varepsilon_{p} E_{3}^{2}\right) \text { for the PZT layer } \\ \frac{1}{2}\left\{\sigma_{m}\right\}^{T}\{S\} \text { for the graphite/epoxy layer. }\end{array}\right.$

Here, for the PZT layer, the first term on the right-hand side is the elastic strain energy of the laminate, the second term is the electromechanical coupling energy and the third term is the electric energy.

Integrating with respect to $z$ in equation (23) results in

$$
\begin{gathered}
\int_{V} H \mathrm{~d} v=\frac{1}{2}\left\{\int_{S}\left[\left(N+N^{p}\right)^{T} \varepsilon^{0}+\left(M+M^{p}\right)^{T} \kappa^{0}\right] \mathrm{d} s\right. \\
\left.+2 \int_{S} V_{3}[e] \varepsilon^{0} \mathrm{~d} s-\int_{S} \varepsilon_{p} \frac{V_{3}^{2}}{h_{p}} \mathrm{~d} s\right\}
\end{gathered}
$$

where $S=a b$ is the area of laminate. Substituting the constitutive equation (14) into (24) results in

$$
\begin{gathered}
\int_{V} H \mathrm{~d} v=\frac{1}{2}\left\{\int_{S}\left(\varepsilon^{0^{T}} A \varepsilon^{0}+2 \kappa^{0^{T}} B \varepsilon^{0}+\kappa^{0^{T}} D \kappa^{0}\right) \mathrm{d} s\right. \\
\left.+2 \int_{S} V_{3}[e] \varepsilon^{0} \mathrm{~d} s-\int_{S} \varepsilon_{p} \frac{V_{3}^{2}}{h_{p}} \mathrm{~d} s\right\} .
\end{gathered}
$$

It is obvious that equation (25) is not only the function of the out-of-plane displacement, $w_{0}(x, y, t)$, and the appled voltage, $V_{3}$, but is also the function of the in-plane displacements $u_{0}(x, y, t)$ and $v_{0}(x, y, t)$. Thus, as explained earlier, it is not feasible to solve such a problem with close-form analytical solutions because, in the cantilever plate we are studying, extension-twisting coupling may cause considerable difficulty in making assumptions about the in-plane solutions to boundary value problems. In order to solve the problem, we use a method mentioned in the book by Whitney [14] and proposed by Reissner and Starsky [15] to rewrite equation (14) in the following way.

By expanding equation (14)

$$
\begin{aligned}
N+N^{p} & =A \varepsilon^{0}+B \kappa^{0} \\
M+M^{p} & =B \varepsilon^{0}+D \kappa^{0}
\end{aligned}
$$

and multiplying the first equation in equation (26) by $\mathrm{A}^{-1}$ yields

$$
\varepsilon^{0}=A^{-1}\left(N+N^{p}\right)-A^{-1} B \kappa^{0} .
$$

Substituting equation (27) into the second term of equation (26) results in

$$
M+M^{p}=B A^{-1}\left(N+N^{p}\right)+\left(D-B A^{-1} B\right) \kappa^{0} .
$$

Letting $A^{*}=A^{-1}, B^{*}=-A^{-1} B$ and $D^{*}=D-B A^{-1} B$ where, in the general case, $A^{*}$ and $D^{*}$ are symmetric while $B^{*}$ is not, equation (14) can be written in the semi-inverted form

$$
\left[\begin{array}{c}
\varepsilon^{0} \\
M+M^{p}
\end{array}\right]=\left[\begin{array}{cc}
A^{*} & B^{*} \\
\left(-B^{*}\right)^{T} & D^{*}
\end{array}\right]\left[\begin{array}{c}
N+N^{p} \\
\kappa^{0}
\end{array}\right]
$$

since $D^{*}=D-B A^{-1} B, D^{*}$ represents a reduction in the bending stiffness of the laminate and $D^{*}$ is referred to as a reduced bending stiffness matrix. Note that none of the structure complexity is lost in the reduced bending stiffness matrix since all elements of the original stiffness matrices contribute.

Now we use the reduced bending stiffness matrix to derive the integration of electric enthalpy of the laminate. Substituting equation (29) into (24), equation (24) becomes

$$
\begin{gathered}
\int_{V} H \mathrm{~d} v=\frac{1}{2}\left\{\int_{S}\left[\left(N+N^{p}\right)^{T} A^{*}\left(N+N^{p}\right)+\kappa^{0^{T}} D^{*} \kappa^{0}\right] \mathrm{d} s\right. \\
\left.+2 \int_{S} V_{3}[e]\left[A^{*}\left(N+N^{p}\right)+B^{*} \kappa^{0}\right] \mathrm{d} s-\int_{S} \varepsilon_{p} \frac{V_{3}^{2}}{h_{p}} \mathrm{~d} s\right\}
\end{gathered}
$$

where $\left(N+N^{p}\right)$ is a function of applied voltage only. According to the plate theory, if the in-plane inertia can be neglected, the equations of motion of the in-plane are

$$
\left\{\begin{array}{l}
\frac{\partial N_{x}}{\partial x}+\frac{\partial N_{x y}}{\partial y}=0 \\
\frac{\partial N_{y}}{\partial y}+\frac{\partial N_{x y}}{\partial x}=0
\end{array}\right.
$$

Expanding $N_{x y}$ in equation (13) results in

$$
N_{x y}=A_{66} \varepsilon_{x y}^{0}+B_{16} \kappa_{x}^{0}+B_{26} \kappa_{y}^{0}-N_{x y}^{0} .
$$

Although there are shearing strains in the graphite/epoxy composite laminae, $\varepsilon_{x y}^{0}=0$, PZT is isotropic in the in-plane and there is no shearing load in the mid-plane of the PZT. Because the laminate only twists, there is no bending and $\kappa_{x}^{0}=0$ and $\kappa_{y}^{0}=0$. As a result, based on equation (32), 
we know that $N_{x y}=0$. Furthermore, based on $N_{x y}=0$ and equations (31), it is derived that $N_{x}$ and $N_{y}$ are respectively constants. According to Saint-Venant's principle, the stress distribution is assumed to be independent of the actual mode of application of the loads except in the immediate vicinity of the point of application of the loads. Furthermore, due to the fact that there are no external forces and moments acting on the laminate, taking a free body of the laminate results in

$$
N_{x}=0 \quad N_{y}=0
$$

The piezoelectric loading $N^{p}$ based on equation (16) is

$$
N^{p}=-V_{3}[e]^{T}
$$

Finally, substituting equations (33) and (34) into equation (30), the integration of electric enthalpy is

$$
\begin{gathered}
\int_{V} H \mathrm{~d} v=\frac{1}{2} \int_{S}\left(\kappa^{0^{T}} D^{*} \kappa^{0}-V_{3}^{2}[e] A^{*}[e]^{T}\right. \\
\left.+2 V_{3}[e] B^{*} \kappa^{0}-\varepsilon_{p} \frac{V_{3}^{2}}{h_{p}}\right) \mathrm{d} s .
\end{gathered}
$$

It is obvious that the integration of the electric enthalpy is only the function of the out-of-plane displacement of the laminate, $w_{0}(x, y, t)$, and the applied voltage, $V_{3}(t)$, and not the function of the in-plane displacements of the laminate, $u_{0}(x, y, t)$ and $v_{0}(x, y, t)$.

\subsection{Equations of motion}

In order to obtain the equations of the motion of the laminate, it is usually an advantage to write the out-of-plane displacement on the mid-plane in the following function:

$$
w_{0}(x, y, t)=\sum_{i} \Phi_{w}^{i}(x, y) p_{w}^{i}(t)
$$

where $w_{0}(x, y, t)$ is the out-of-plane displacement on the mid-plane of the laminate, $\Phi_{w}(x, y)$ is the vibrational shape function that satisfies the boundary condition of the laminate, $p_{w}(t)$ is the generalized out-of-plane displacement variable that is a function of time, and the superscript $i$ denotes the $i$ th order vibration mode (omitting the letter $i$ in $\Phi_{w}^{i}$ and $p_{w}^{i}$ in the following).

For the laminate, the mass force $G$ and the traction $f$ in equation (3) are zero. If the in-plane inertial terms can be neglected, substituting equations (5) and (35) into equation (3), considering equation (36) and carrying out the variation of the generalized out-of-plane displacement $p_{w}(t)$ and the applied voltage $V_{3}(t)$ result in the equations of the motion of the laminate. For the $i$ th order vibration mode, the governing equations of motion of the laminate are

$$
\begin{aligned}
M_{i} \ddot{p}_{w}(t)+C_{i} \dot{p}_{w}(t)+K_{i} p_{w}(t) & =\Theta_{e i} V_{3}(t) \\
C_{e} V_{3}(t)+\Theta_{e i} p_{w}(t)+Q_{e} & =0
\end{aligned}
$$

where

$$
\left\{\begin{aligned}
M_{i}= & \int_{s} \rho \Phi_{w}^{2} \mathrm{~d} s \\
C_{i}= & 2 M_{i} \omega_{n} \xi_{i} \\
K_{i}= & \int_{s}\left[D_{11}^{*}\left(\frac{\partial^{2} \Phi_{w}}{\partial x^{2}}\right)^{2}+2 D_{12}^{*} \frac{\partial^{2} \Phi_{w}}{\partial x^{2}} \frac{\partial^{2} \Phi_{w}}{\partial y^{2}}\right. \\
& \left.\quad+D_{22}^{*}\left(\frac{\partial^{2} \Phi_{w}}{\partial y^{2}}\right)^{2}+4 D_{66}^{*}\left(\frac{\partial^{2} \Phi_{w}}{\partial x \partial y}\right)^{2}\right] \mathrm{d} s \\
\Theta_{e i}= & \int_{s} 2 e_{31}\left(B_{16}^{*}+B_{26}^{*}\right)\left(\frac{\partial^{2} \Phi_{w}}{\partial x \partial y}\right) \mathrm{d} s \\
Q_{e}= & \int_{s} q_{3} \mathrm{~d} s \\
C_{e}= & \int_{s}\left[e_{31}^{2}\left(A_{11}^{*}+2 A_{12}^{*}+A_{22}^{*}\right)+\frac{\varepsilon_{p}}{h_{p}}\right] \mathrm{d} s
\end{aligned}\right.
$$

in which

$$
\begin{array}{r}
\rho=\int_{-h / 2}^{h / 2} \rho_{0}^{(k)} \mathrm{d} z \\
\omega_{n}=\sqrt{K_{i} / M_{i}}
\end{array}
$$

where $M_{i}, K_{i}$ and $C_{i}$ represent the mode mass, mode stiffness and coefficient of mode damping of the laminate, respectively. Note that $M_{i}, K_{i}$ and $C_{i}$ are relative to boundary conditions, and $\xi_{i}$ is the damping factor. Also note that the damping term is artificially added. $\omega_{n}$ is the natural frequency of the laminate. The term $\Theta_{e i}$ represents the electromechanical coupling and describes the conversion of the applied voltage to an equivalent force on the laminate. It is therefore called the electromechanical coupling factor. $Q_{e}$ denotes the electric charge produced on the piezoelectric layer. $q_{3}$ denotes the electric charge per unit area produced on the electrode. $C_{e}$ denotes the electric capacity of the piezoelectric layer. It should be noted that equation (37) is the equation of motion and equation (38) is the sensor equation of the laminate.

\subsection{Solution of equations}

We consider the solution of equations (37) and (38) when the applied voltage is expressed in the form

$$
V_{3}(t)=V_{3 m} \sin \omega t
$$

where $V_{3 m}$ and $\omega$ are the amplitude and the frequency of the applied voltage, respectively.

We let

$$
\begin{aligned}
r & =\frac{\omega}{\omega_{n}} \\
P_{w} & =\frac{V_{3 m} \Theta_{i}}{K_{i}}
\end{aligned}
$$

where $r$ is the ratio of the frequency of the applied voltage to the natural frequency of the laminate, and $P_{w}$ the static generalized out-of-plane displacement of the laminate. The steady-state solutions $p_{w}(t)$ and $Q_{e}$ of equations (37) and (38) are

$$
\begin{gathered}
p_{w}(t)=P_{w} \beta \sin (\omega t-\varphi) \\
Q_{e}=-C e V_{3 m} \sin \omega t-\Theta_{i} P_{w} \beta \sin (\omega t-\varphi)
\end{gathered}
$$

where $\beta$ is the magnification factor defined in the case of damped systems as

$$
\beta=\frac{1}{\sqrt{\left(1-r^{2}\right)^{2}+\left(2 r \xi_{i}\right)^{2}}} .
$$


Table 1. Material properties of piezoelectric and composite materials.

\begin{tabular}{lll}
\hline Material properties & Graphite/Epoxy & Piezoelectric material \\
\hline$E_{1}(\mathrm{GPa})$ & 138 & 61 \\
$E_{2}(\mathrm{GPa})$ & 8.96 & 61 \\
$G_{12}(\mathrm{GPa})$ & 7.1 & 23.3 \\
$v_{12}$ & 0.3 & 0.31 \\
$\rho\left(\mathrm{kg} \mathrm{m}^{-3}\right)$ & 1560 & 7500 \\
$d_{31}\left(\mathrm{~m} \mathrm{~V}^{-1}\right)$ & & $-171 \times 10^{-12}$ \\
$\varepsilon_{p}\left(\mathrm{~F} \mathrm{~m}^{-1}\right)$ & & $1.65 \times 10^{-8}$ \\
$a \times b \times h_{c}(\mathrm{~mm})$ & $25 \times 90 \times 1$ & \\
$a \times b \times h_{p}(\mathrm{~mm})$ & & $25 \times 90 \times 1$ \\
\hline
\end{tabular}

The physical meaning of $\beta$ is the ratio of the amplitude of dynamic displacement to static displacement. $\varphi$ is the phase angle defined by

$$
\varphi=\tan ^{-1}\left(\frac{2 r \xi_{i}}{1-r^{2}}\right)
$$

As a result, the out-of-plane displacement of the laminate in the steady state is

$$
w(x, y, t)=\Phi_{w}(x, y) P_{w} \beta \sin (\omega t-\varphi) .
$$

It should be noted that the vibration of the laminate is the sum of all vibration modes. However, depending on the excitation frequency near the natural frequency of the laminate, only a few eigenmodes are excited. Therefore, only the corresponding eigenmode needs to be considered and all others may be neglected. As a result, we obtain the solution of the equations of motion of the laminate with a very simple form.

\section{Results and discussion}

In the present study, a three-layer sandwiched plate, as shown in figure 1, is used for demonstration. The top and bottom layers are the graphite/epoxy composite with orientation $\pm 45^{\circ}$. The center layer is PZT. The composite lamina is Hercules AS4/3501-6 graphite/epoxy and the piezoelectric material is Morgan Matroc PZT-5H. The material properties of the lamina shown in figure 1 are listed in table 1. The PZT plate is coated with a silver electrode at its two largest surfaces. The dimensions of the PZT plate are $90 \times 25 \times 1 \mathrm{~mm}^{3}$. The PZT is sandwiched between two graphite/epoxy laminated plates with the dimensions of $90 \times 25 \times 1 \mathrm{~mm}^{3}$.

In order to use the proposed analytical model to obtain the numerical results of the laminate, first we introduce a shape function, $\Phi_{w}(x, y)$, which leads to an approximate solution of the excited eigenmode vibration. For the torsional vibration, we can find the following shape function in the separatevariable form:

$$
\Phi_{w}(x, y)=X_{0}(x) Y_{0}(y)
$$

where

$$
\begin{gathered}
X_{0}(x)=\sin \eta x-\sinh \eta x+D(\cos \eta x-\cosh \eta x) \\
D=\frac{\cos \eta a+\cosh \eta a}{\sin \eta a-\sinh \eta a} \\
\eta a=1.875 \\
Y_{0}(y)=y .
\end{gathered}
$$

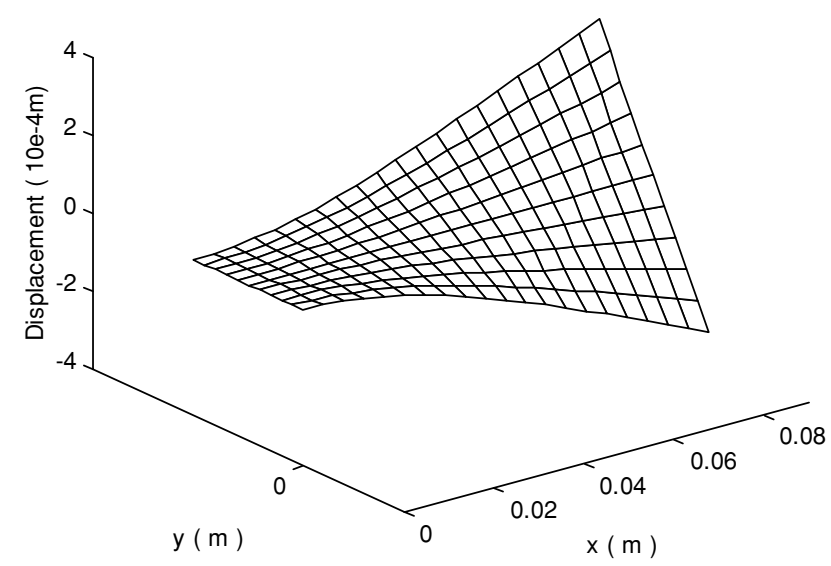

Figure 2. Mode shape of the torsional vibration.

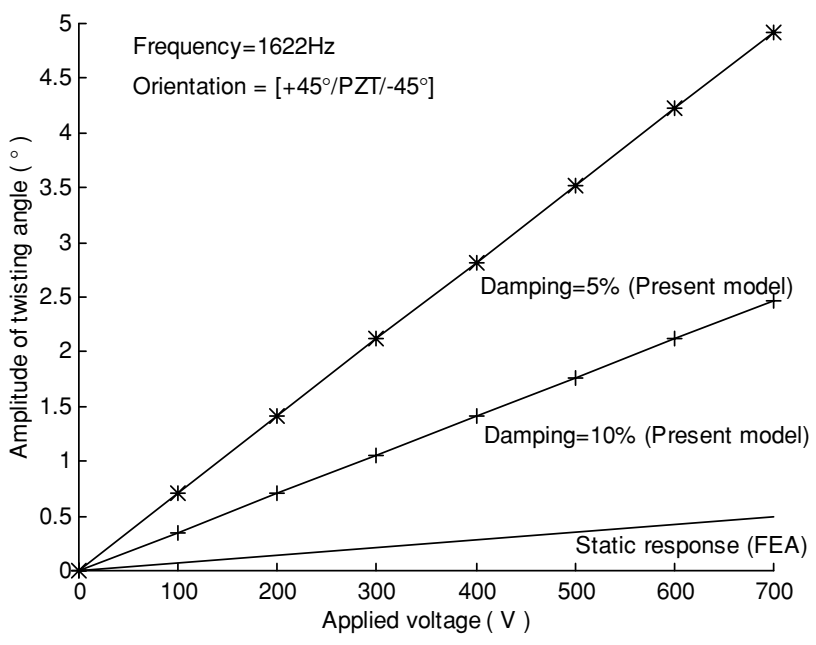

Figure 3. Amplitude of twisting angle of the laminate excited at different applied voltages with various damping factors.

$X_{0}(X)$ and $Y_{0}(y)$ are assumed to be vibration shape functions in the $x$ - and $y$-directions, respectively, which satisfy the boundary conditions of the fixed-free plate. $\eta a=1.875$ is obtained from a solution of the characteristic equation of transverse vibration of the fixed-free plate strip.

A commercial code MATLAB is used to compile the analytical programs for simulation. The torsional vibration of the plate was simulated, and the displacement of one of the two corners at the free end was used to represent the responses. Therefore, the twisting angle of the laminates can be calculated by dividing this displacement by the half width of the laminate. Furthermore, in order to confirm the proposed analytical model, comparisons between the numerical results obtained from the proposed model and FEA were made. The FEA result uses the ANSYS software and uses an eight-node brick element.

The numerical results of the natural frequency of the laminate between the proposed model and the FEM for the torsional vibration, as shown in figure 2, are $1622 \mathrm{~Hz}$ and $1616 \mathrm{~Hz}$, respectively. From these results, we know that the result obtained by the developed model and FEA agree well. Figure 3 shows the amplitudes of the twisting angle of the 


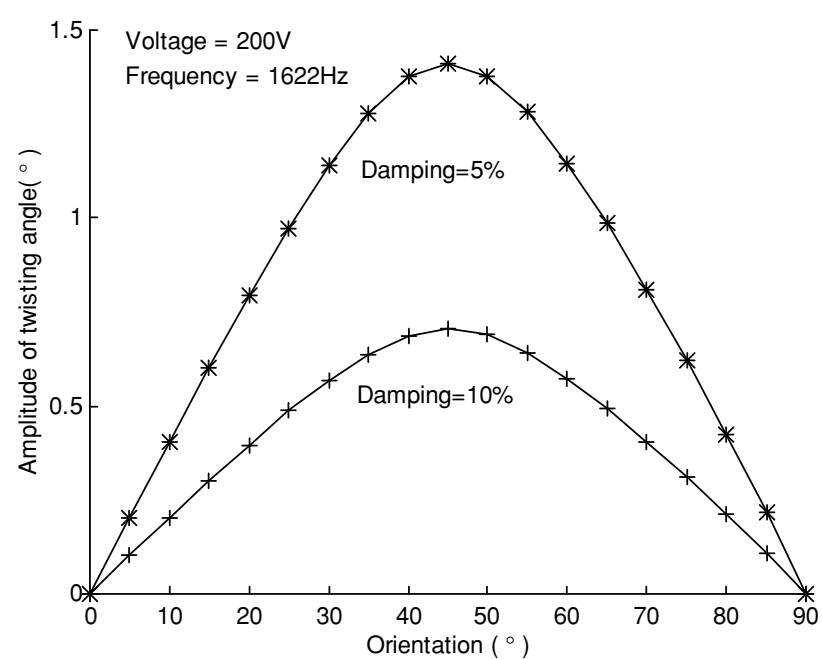

Figure 4. Relationship between amplitude of twisting angle and fibre orientation.

laminate excited at different applied voltages with two different damping factors, $5 \%$ and $10 \%$. According to vibration theory, it is known that, for $\omega=\omega_{\mathrm{n}}$, the magnification factor $\beta$ is equal to $1 / 2 \xi_{i}$ for a damped system. From this point of view, if $\xi_{i}$ is equal to $5 \%$, the amplitude of the dynamic twisting angle is ten times that of the static twisting angle for the same input voltage condition; if $\xi_{i}$ is equal to $10 \%$, the dynamic twisting angle is five times that of the static twisting angle. It is observed that the analytic results and the FEA static results also agree very well in this respect, where the FEA static response data are taken from [10]. Moreover, it is also clearly observed that the amplitude of twisting angle is highly affected by the damping. Therefore, in order to obtain a large amplitude of twisting angle, we need a small damping factor for the laminate.

It is known that torsional vibration is produced by extension-twisting coupling. When the orientation is equal to $\left[0^{\circ} / \mathrm{PZT} / 0^{\circ}\right]$ or $\left[90^{\circ} / \mathrm{PZT} /-90^{\circ}\right]$, because $\mathrm{B}_{16}^{*}$ and $\mathrm{B}_{26}^{*}$ are equal to zero, there is no extension-twisting coupling. When the orientation is equal to $\left[45^{\circ} / 0^{\circ} /-45^{\circ}\right]$, the values of $\mathrm{B}_{16}^{*}$ and $\mathrm{B}_{26}^{*}$ are maximized. As a result, this orientation $\left[45^{\circ} / 0^{\circ} /-45^{\circ}\right]$ leads to the maximum amplitude of twisting angle of the laminates. Figure 4 shows the relationship between the amplitudes of the twisting angle and the fibre orientation of laminae. The results shown in figure 4 support the actuation principle of the torsional vibration of extensiontwisting coupling and also support the design of orientation $\left[45^{\circ} / \mathrm{PZT} /-45^{\circ}\right]$ for a rotary motor driven by an anisotropic piezoelectric composite laminated plate [12].

Figure 5 shows the numerical results of the frequency responses of the laminate with the configuration of orientation $\left[45^{\circ} / \mathrm{PZT} /-45^{\circ}\right]$ using the developed model, where the driving voltage is $200 \mathrm{~V}$.

\section{Conclusions}

In this paper we present a dynamic analytical model of torsional vibration induced by the extension-twisting coupling of anisotropic composite laminates with piezoelectric

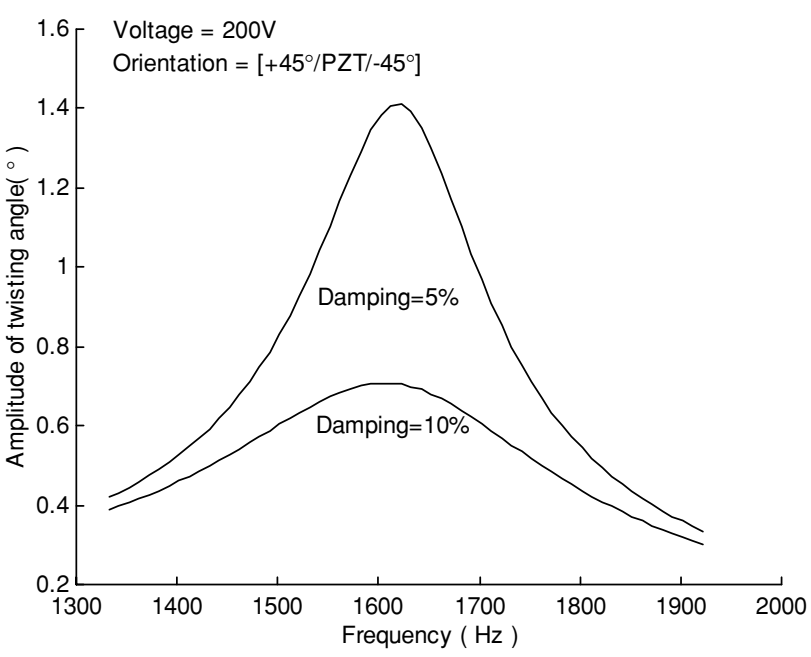

Figure 5. Frequency response of $\left[+45^{\circ} / \mathrm{PZT} /-45^{\circ}\right]$ excited at $200 \mathrm{~V}$.

actuators using a non-conventional method (a reduced bending stiffness matrix). By using the matrix, the integration of electric enthalpy has been expressed as only the function of the out-of-plane displacement of the laminate, $w_{0}(x, y, t)$, and the applied voltage, $V_{3}(t)$, and not as the function of the in-plane displacements of the laminate, $u_{0}(x, y, t)$ and $v_{0}(x, y, t)$. Thus, the method changes a three-dimensional problem into a one-dimensional problem, and none of the structure complexity is lost because all elements of the original stiffness matrices contribute. Finally, the model allows the analysis of anisotropic plate to be directly utilized. We can say that the modeling supplies a basis for analyzing some coupling vibration problems. In addition, the Hamilton principle and the classical plate theory are used for the derivation of the model. We have given the analytical solution of the model in the case of harmonic excitation. We have presented some comparisons with a more accurate FEA model. The analytical model is verified by finite element analysis. The analytical model has the ability to predict the dynamic characteristics and to explain the actuation mechanism of the twisting motion of piezoelectric composite laminates.

\section{Acknowledgments}

The Research Grant Council of Hong Kong has sponsored this study through the grant HKUST6050/97E to the Hong Kong University of Science and Technology (HKUST). The authors wish to acknowledge this support. M-L Zhu also wishes to acknowledge the support of the Alexander von Humboldt Foundation during the revising process.

\section{References}

[1] Sunar M and Rao S 1999 Recent advances in sensing and control of flexible structures via piezoelectric materials technology Appl. Mech. Rev. 521

[2] Saravanos D A and Heyliger P R 1999 Mechanics and computational models for laminated piezoelectric beams, plates, and shells Appl. Mech. Rev. 52305 
[3] Tani J, Takagi T and Qiu J 1998 Intelligent material systems: Application of functional materials Appl. Mech. Rev. 51505

[4] Aldraihem O J and Wetherhold R C 1997 Mechanics and control of coupled bending and twisting vibration of laminated beams Smart Mater. Struct. 6123

[5] Dimitriadis E K, Fuller C R and Rogers C A 1991 Piezoelectric actuators for distributed vibration excitation of thin plate J. Vib. Acoust. 113100

[6] Wang B T and Rogers C A 1991 Modeling of finite-length spatially distributed induced strain actuators for lamina beams and plates J. Intell. Mater. Syst. Struct. 238

[7] Shen Y 1995 Bending and torsional vibration control of composite beams through intelligent constrained-layer damping treatments Smart Mater. Struct. 4340

[8] Yang S M and Bian J J 1996 Vibration suppression experiments on composite laminated plates using an embedded piezoelectric sensor and actuator Smart Mater. Struct. 5501

[9] Park C, Walz C and Chopra I 1996 Bending and torsion models of beams with induced-strain actuators Smart Mater. Struct. 598
[10] Lee S-W R and Chan K-H W 1995 Actuation of torsional motion for piezoelectric laminated beams Dynamic response and behavior of composites ASME AD-46 pp 139-46

[11] Lee S-W R and Sun C T 1995 Bending/twisting/shearing actuation and sensing of laminated composite beams with piezopolymer film Intell. Mater. Syst. 137

[12] Lee S-W R and Li H L 1998 Development and characterization of a rotary motor driven by anisotropic piezoelectric composite lamina Smart Mater. Struct. 7327

[13] Lee S-W R and Li H L 1998 Torsional vibration control of piezoelectric composite laminate using structure coupling Proc. 4th Int. Conf. on Intelligent Materials (Chiba, Japan) pp 158-59

[14] Whitney J M 1987 Structural Analysis of Laminated Anistropic Plates (Lancaster, PA: Technomic Publishing Company)

[15] Reissner E and Starsky Y 1961 Bending and stretching of certain types of heterogeneous aerolotropic elastic plates J. Appl. Mech. 28402

[16] Parton V Z and Kudryavtsev B A 1988 Electromagnetoelasticity (New York: Gordon and Breach) 